\title{
Research on the Setting of Australian Mountain Fire Emergency Center Based on $K$-Means Algorithm
}

\author{
Chenyang Tang $\mathbb{D},{ }^{1}$ Hanwen Zhang, ${ }^{2}$ Songze Liu, ${ }^{2}$ Guanlin $\mathrm{Zhu},{ }^{3}$ Minghao Sun, \\ Yushuai $W u \mathbb{D}^{1,4}$ and Yongde Gan ${ }^{1}$ \\ ${ }^{1}$ School of Water Resources and Electric Power, Qinghai University, Xining 810016, China \\ ${ }^{2}$ School of Computer Science, Qinghai University, Xining 810016, China \\ ${ }^{3}$ School of Ecological Environment Engineering, Qinghai University, Xining 810016, China \\ ${ }^{4}$ Key Laboratory of Ecological Protection and High-Quality Development in the Upper Yellow River, Xining, \\ Qinghai Province, China \\ Correspondence should be addressed to Yushuai Wu; ys.wu@qhu.edu.cn
}

Received 13 April 2021; Revised 1 August 2021; Accepted 18 August 2021; Published 14 September 2021

Academic Editor: Zeshui Xu

Copyright (c) 2021 Chenyang Tang et al. This is an open access article distributed under the Creative Commons Attribution License, which permits unrestricted use, distribution, and reproduction in any medium, provided the original work is properly cited.

\begin{abstract}
The Australian wildfires in 2019-2020 have brought suffering to the Australian people. It is essential to use models to help the Victorian government monitor and predict the occurrence and development of fires to the greatest extent possible under the principles of safety and economy to facilitate rapid response. Through the idea of $K$-means algorithm and greedy algorithm, we, respectively, analyzed cities and rural areas at different altitudes and combined the altitude with the obtained clusters; the analysis from the established model shows that, for cities, cluster areas with smaller clusters with an altitude of less than 1600 meters and areas with smaller clusters with an altitude of greater than or equal to 1800 meters are covered by an EOC; for areas with larger clusters less than or equal to 600 meters above the sea level and areas with larger clusters greater than or equal to 1000 meters above the sea level, we use two EOCs for coverage; for rural areas, all areas with smaller clusters are covered by one EOC, while for areas with larger clusters where the altitude is less than or equal to 1000 meters and the altitude is greater than or equal to 1600 meters, we use two EOCs for coverage; also, obtained through greedy algorithm analysis, one EOC corresponds to 14 SSA UAVs and 8 repeater UAVs, and two EOCs correspond to 12 repeater UAVs and 26 SSA UAVs. We have a reason to believe that, through our mathematical model and the leaps in drone technology, it will have a long-term and profound impact on Australia's wildfire control.
\end{abstract}

\section{Introduction}

Forest fire refers to forest fire behavior that is beyond human control; spreads and expands freely in forest land; and brings certain harm and loss to forests, forest ecosystems, and humans. It is a natural disaster that is sudden, destructive, and difficult to handle and salvage [1]. It is a kind of fire that occurs during the seasonal alternation of severe dryness and continuous high temperature and is the main contributor to the active gas phase and particulate phase organic compounds in the atmosphere [2]. As climate warming intensifies, the frequency and intensity of global forest fire disasters will also increase. The potential dangers of forest fires worldwide will exist for a long time, and the severity will increase significantly [3], causing severe impacts on the global ecological environment [4].

Australia is the country with the largest area in Oceania and the country with the most severe wildfire problem. On average, there are more than 50,000 wildfires every year, and about 50 million $\mathrm{hm}^{2}$ of forest and grassland are destroyed by wildfires [5]. The disastrous forest fires in Australia from October 2019 to February 2020 have made humankind more clearly aware of the enormous impact of wildfires on the environment and climate. In response to wildfires' problem, 
Australian fire fighters currently use remote-controlled aircraft surveillance and situational awareness [6] (SSA) that carry high-definition cameras with red heat detection capabilities and remote sensing sensors to monitor and sense the fire situation for emergency use. Quick response monitoring of the Operation Center (EOC) [7]. The Emergency Operations Center is a place for responding to public health emergencies in medicine [8]. Similarly, it is also a place for responding to sudden natural disasters. Through the real-time monitoring capabilities of the SSA UAV, the EOC and the SSA's monitoring capabilities are matched, and the best way to command the areas with hidden dangers is to dispatch reasonable personnel to eliminate forest fires in the cradle. [9].

The communication of two-way wireless walkie-talkies allows the "ground operations" frontline team to provide status reports to EOC and enables EOC (Emergency Operations Center) to frontline team's work. Rural areas mainly depend on distance and terrain, and in urban areas, it mainly depends on the occlusion of buildings [10]; repeaters are used as a transceiver that automatically rebroadcasts signals with the higher power to expand the radio range and is generally used in the microwave front end of the UAV [11], the repeater located between the front line and the EOC can relay the radio signal from the front line to the EOC, or from the EOC to the front line. At present, most of the bee colony algorithms are used in UAV fire-fighting mission planning [12]; similarly, in the process of multi-UAV mission planning, Deng et al. [13] and others introduced an improved quantum particle swarm algorithm to carry out tasks. Planning; Kurdi et al. [14] designed a locust bionic algorithm to simulate fire development uncertainly, but these algorithms are limited to traditional single-constraint problems and cannot solve practical problems with multiple constraints. The universality is very poor.

At present, more and more countries use UAV monitoring systems as an important method to prevent forest fires [15]. However, how to ensure the UAV monitoring system's economy and the integrity of the monitoring range is an important problem currently existing in the UAV monitoring system. Reddy et al. [16] collected daily MODIS data on fire sites in Australia from 2005 to 2020, combined with geospatial technology to study forest fires' spatial and temporal patterns. Cawson and Duff [17] and others established a logistic regression model using combustion data to predict the fire probability of combustible bed at the time and space scale and determine the influence mechanism of different properties and flammability of combustible bed under extreme weather conditions. Almeida et al. [18] studied the burning characteristics of eucalyptus bark based on the azimuth angle $\theta$ of burning particles related to the airflow and the flow velocity $U$ around the particles. They evaluated the burning characteristics of eucalyptus bark and proposed a fire spread empirical model to simulate the impact of wildfires on forests. Thus, through the analysis of these mechanisms, combined with drones' existing characteristics, real-time monitoring of the common wildfires in Australia can be carried out.

\section{Method}

2.1. Clustering Algorithm. Clustering analysis is composed of several patterns based on similarity. There are more similarities between patterns in a cluster than patterns that are not in the same cluster [19]. Usually, a pattern is a metric vector or a point in a multidimensional space; cluster analysis, as one of the commonly used methods in traditional machine-learning algorithms, is widely favored due to its practical, efficient, and straightforward characteristics [20]. Typical clustering algorithms are divided into three stages: feature selection and feature extraction, calculation of similarity between data objects, and grouping of data objects according to similarity [21]. Data objects are divided into multiple classes or clusters. Objects in the same cluster have a high degree of similarity, but objects in different clusters are quite different [22].

Clustering algorithms can be divided into hierarchical clustering algorithms and partitioned clustering algorithms [23]. The hierarchical clustering algorithm attempts to build a high-level nested clustering tree structure through data objects' similarity between different categories. The clustering trees' construction includes agglomerated hierarchical clustering (building a tree structure in a bottom-up manner) and splitting-type hierarchical clustering (build tree structure from top to bottom). The partition clustering algorithm should first specify the number of clusters and cluster centers and divide the data set into several disjoint clusters by optimizing some loss functions, in order to simplify the model by analyzing different clusters.

2.2. Greedy Algorithm. Greedy algorithm, also known as a greedy method, is an improved hierarchical processing method [24], and is a standard method to find the optimal solution; it is always making the current optimal choice; in other words, the greedy algorithm is not considered from the whole, but is the optimal choice in a specific local sense [25]. The basic idea of the greedy algorithm is to start from a sure initial solution of the problem and gradually approach the given goal in order to find a better solution as quickly as possible [26] to find the optimal local solution in the whole, and all these local optimal solutions combine to form an optimal solution as a whole [27]. Compared with the general scheduling algorithm, the greedy algorithm has simple algorithms, low complexity, and high efficiency [28]. It is a standard and excellent method to solve the problem of better choice [29].

The key feature of the greedy algorithm is to adopt a selection strategy with no aftereffects. The previous state only depends on the existing state and will not affect the subsequent process $[30,31]$; it generally includes the following four steps: first, select optimization greedy standard. Second, verify that this problem can meet the conditions of greedy selectivity and optimal substructure under the above criteria. Third, implement data sorting according to the greedy metric, determine the greedy selection algorithm, and solve a subset of feasible solutions. Fourth, calculate the optimal solution according to the greedy sequence and the objective function. 


\section{Implementation Methods}

3.1. Data Processing and Assumptions. For the monitoring of wildfires in the Victoria area, we considered the EOC location and established the optimal location model through the clustering algorithm. At the same time, in order to consider the number of drones used under the most basic and ideal conditions, we simulated the best combination of zero-height plane direct drones and SSA drones, and on this basis, the zero-height optimal coverage model is established. Based on the obtained results, we further estimated the number of drones required on Victoria's ground. We combined these two models to get a quick emergency response model. At the same time, in order to make the model closer to the actual situation, we have considered some practical factors. They are: (1) the detection range of the equipment worn by the detector; (2) the detection range of the UAV; and (3) the influence of local climatic conditions and terrain conditions. Similarly, in the process of UAV forest fire detection, the following assumptions are made for analysis: (1) communication equipment and monitoring equipment do not consider the impact of day and night and extreme weather; (2) the monitoring range of SSA UAV is not affected by terrain factors; (3) the battery life is not considered to decrease with use; and (4) the visual inspection range of the human body to the fire is two kilometers.

When analyzing team performance, the data used in this model mainly are from complete data provided by the Federation of Mathematics and Its Applications (COMAP). Besides, we have inquired about other firerelated data from https://www.kaggle.com/carlosparadis/ fires-from-space-australia-and-new-zeland and https:// www.peakbagger .com/range.aspx? rid $=724$ to obtain fire point data for the Australian fire season. After filtering Victoria's latitude and longitude, the fire location, fire intensity, and fire frequency of the state are obtained. At the same time, we obtained terrain data for Australia in Figure 1.

Besides, we obtained the topographic map and elevation data of the area clearly through the surveying and processing of ArcGIS software. Furthermore, the data are shown in Figure 2:

Based on the above data, we continue to carry out further data processing. We learned that on the same meridian, 1 degree of latitude is about 111 kilometers, expressed as $D_{2}$. In latitude, for every 1-degree difference in longitude, the actual distance is $111 * \cos \theta \mathrm{km}$, expressed as $D_{1}$. In addition, according to the drone's known parameters given in the title, we can find that the farthest flying distance of the drone is 30 kilometers; that is, the radius of the circle centered on the EOC is 30 kilometers. It can be seen from the above that the diameter of each circle centered on EOC is 60 kilometers, and two EOC circles can cover a line of latitude. From the drawn map, we can see that there is no fire covering more than two latitude lines, so the formula is as follows:

$$
\begin{aligned}
& D_{1}=111 * \cos \theta, \\
& D_{2}=111 .
\end{aligned}
$$

3.2. Model Establishment and Analysis Based on K-Means Algorithm. K-means algorithm, as the most popular algorithm in clustering algorithms, is an iterative process [32]; it was first used by MacQueen [33] in 1967. Compared to other clustering algorithms, the $K$-means algorithm has been widely used in clustering algorithms due to its better effect and simple idea. Similarly, it is also unsupervised learning and a clustering algorithm based on partition [34]. Euclidean distance is generally used as an index to measure the similarity between data objects. The similarity is inversely proportional to the distance between data objects. The algorithm needs to specify the number of initial clusters $k$ and $k$ initial cluster centers in advance. According to the similarity between the data objects and the cluster centers, the positions of the cluster centers are constantly updated. The sum of squared errors of the clusters is continuously reduced (Sum of Squared Error, SSE) when the SSE no longer changes or the objective function converges, the clustering ends, and the final result is obtained.

The core idea of the $K$-means algorithm is: first randomly select $k$ initial cluster centers $C_{i}(1 \leq i \leq k)$ from the data set, calculate the Euclidean distance between the remaining data objects and the cluster center $C_{i}$, and find the distance; the closest cluster center $C_{i}$ of the target data object and the data object are allocated to the cluster corresponding to the cluster center $C_{i}$. The average value of the data objects in each cluster is calculated as the new cluster center, and the next iteration is performed until the cluster center no longer changes or the maximum number of iterations stops.

The formula for calculating the Euclidean distance between the data object and the cluster center in space is

$$
d\left(x, C_{i}\right)=\sqrt{\sum_{j=1}^{m}\left(x_{j}-C_{I J}\right)^{2}} .
$$

Among them, $x$ is the data object, $C_{i}$ is the $i$ th cluster center, $m$ is the dimension of the data object, and $x_{j}$ and $x_{j}$ are the $j$-th attribute values of $x$ and $C_{i}$, respectively.

The formula for calculating the sum of squared errors SSE of the entire data set is

$$
\mathrm{SSE}=\sum_{i=1}^{k} \sum_{x \in C_{1}}\left|d\left(x, C_{i}\right)^{2}\right| .
$$

Among them, the size of SSE indicates the clustering result's quality, and $k$ is the number of clusters.

In the traditional $K$-means algorithm, the number of clusters $k$ needs to be determined in advance, but in practice, it is often difficult to determine the value of $k$ due to a large amount of data and lack of experience. If the value of $k$ selected is too small, it will result in the same cluster. The data objects are very different. If the $k$ value selected is too large, the difference between different clusters will be slight. To avoid manually selecting the value of $k$, we use the hierarchical $k$-means clustering algorithm [35]. At the beginning of the algorithm, just like the traditional $K$-means algorithm, $k$ initial clustering centers are randomly selected 


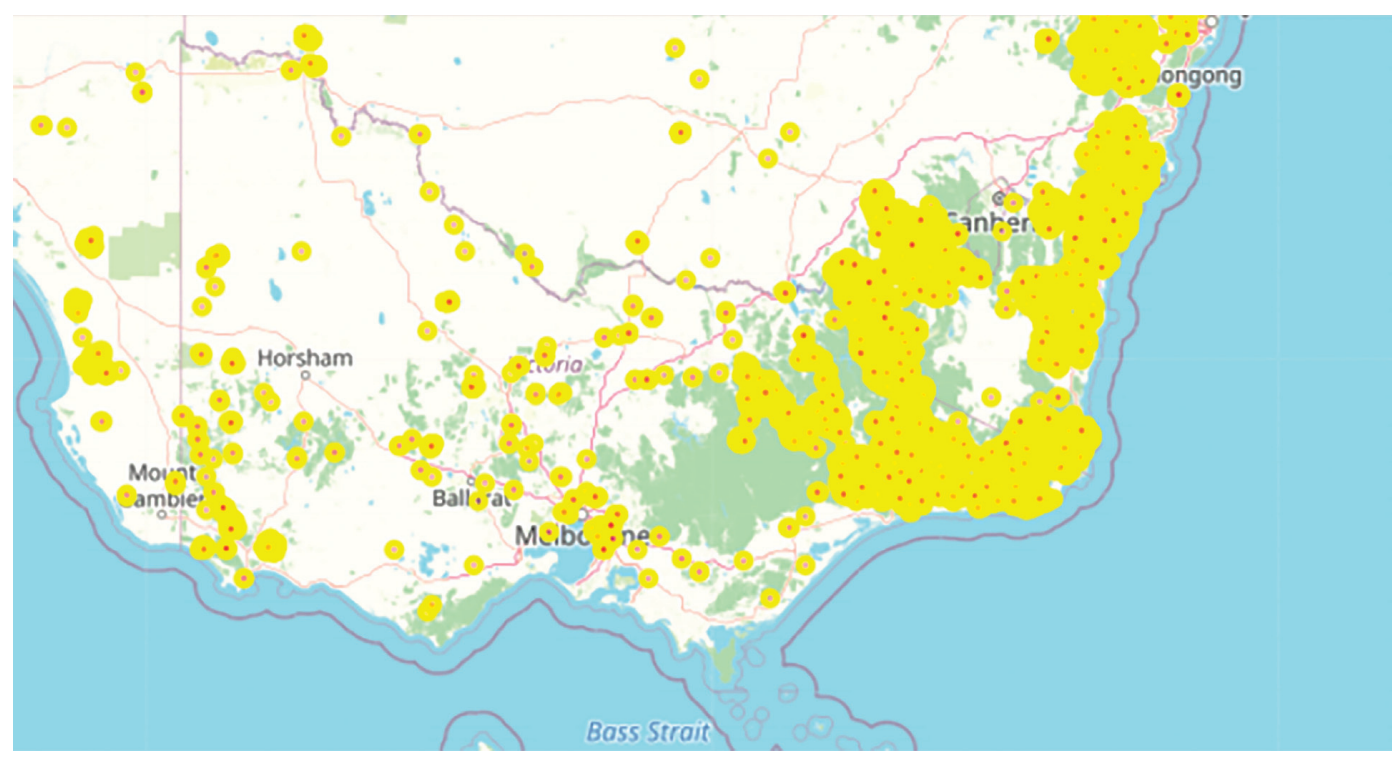

Figure 1: Topographic data of Australian fire spots.



FIGURE 2: Australia topographic map. 
for one iteration, and then the clustering is calculated. The clustering measures are as follows:

$$
J=\sqrt{\frac{\sum_{i=1}^{k} \sum_{j=1}^{n_{i}}\left(x_{i j}-c_{i}\right)^{2}}{n-1} .}
$$

Perform a finer level of clustering. $C_{i}$ is the cluster center of the $i$ th class, $x_{i j}$ is the $j$ th data object in the $i$ th class, $n_{i}$ is the number of data objects in the $i$ th class, $k$ is the number of clusters, and $n$ is data set size. After each iteration, the cluster center finds the cluster with the largest radius among all clusters, selects the two most distant sample points in the cluster as the new cluster center, and reiterates with other cluster centers. Calculation of the ratio of clustering measures after each iteration:

$$
\varepsilon=\frac{J^{(t)}-J^{(t-1)}}{J^{(t-1)}} .
$$

Among them, $t$ is the number of iterations. If $\varepsilon$ is greater than the artificially set empirical value $\Delta$, clustering is continued; otherwise, the algorithm stops, and the clustering result and the optimal number of clusters $k$ are output. The results show that compared with the clustering accuracy of the $K$-means algorithm, the clustering accuracy is greatly improved. The algorithm can also obtain $k$ values close to the actual number of clusters. Based on the above steps, we can get the following results through clustering in Figure 3:

As shown in Figure 3, the clusters are densely distributed in eastern Victoria, indicating that eastern Victoria is an area with frequent wildfires. For this area, more EOCs should be established, and more drone equipment should be purchased. In western and central Victoria, the clusters are sparsely distributed, indicating that wildfires in these areas are not very frequent, but only occasionally; therefore, the EOC can be set appropriately, but the purchase of drone equipment still needs to increase to avoid risks as much as possible.

Due to Victoria's complex terrain, the altitude represented by each cluster center is different, so a basic platform is needed to conduct corresponding research on cluster centers in various regions. On this basis, we simplified the altitude of various locations in Victoria according to the ideal state and regarded the entire Australian continent as the zero-altitude point. When analyzing a specific location, we multiplied the actual altitude by our assumption that every increase of 100 meters will reduce the communication distance by 500 meters, thereby simulating the actual communication distance:

$$
\left\{\begin{array}{l}
l^{\prime}=l-5 h, \\
h_{(0)}=0, \\
h-h_{(0)}=h_{v} .
\end{array}\right.
$$

Among them, $l$ is the communication distance at zero altitude under normal conditions, $l^{\prime}$ is the actual communication distance as the altitude rises, $h$ is the altitude, $h_{(0)}$ is the zero altitude, $h_{v}$ is the simplified Victorian area altitude, the value is the same as $h$. So we can get the changing trend of the range that the repeater drone can cover with altitude in Figure 4 .

\section{Results and Discussion}

Therefore, after we simplify the entire state of Victoria, Australia, our goal is to establish facilities that can cover all points and meet the most economical requirements for areas with high fire hazards. In other words, we need a minimum number of direct drones to cover all potential fire points in order to achieve the purpose of regular communication between wearable equipment personnel and EOC.

First of all, we analyze the maximum number of drones placed in a cluster; too many drones will cause waste of resources, which will increase costs needlessly; too few drones will make it challenging to complete monitoring potential fire points so that the fire point cannot be alerted the first time. Therefore, the cluster analysis we get is shown in Figure 5:

As shown in the figure, the red circle represents the effective detection range of the SSA, the blue circle represents the communication signal range of the repeater drone, each blue circle represents a repeater drone, and each red circle represents an SSA; strive to solve the problem of the number of UAV transponders. Our goal is to achieve the largest monitoring area based on saving costs as much as possible. The number of drones is a direct manifestation of the cost. Simultaneously, according to the performance of drones, we can make them work in different areas to achieve the maximum coverage effect.

As can be seen from the above figure, in the idealized Victorian area, it is the most economical for us to use three direct drones simultaneously, and the coverage is also relatively comprehensive. At the same time, it can also adapt well to the harmful effects of different terrain changes. When the altitude increases, we can achieve comprehensive condition coverage by increasing the minimum number of repeater drones, which guarantees safety and conforms to economic principles. No more economic loss. Since the repeater drones need to work in turns, and the charging time is often less than the flight time, theoretically speaking, the drone still needs a standby drone to perform monitoring tasks when the power is exhausted and return home to achieve a 24-hour uninterrupted cycle for detection; the total number of patrol drones should be twice the number of drone patrols required by the cluster. Similarly, for safety and possible failure considerations and some areas with higher fire potential hazards, we will once again increase the number of drones with one-third of the total number of patrol drones as backups. In this way, more comprehensive coverage can be carried out in places with severe fire conditions. At the same time, it can also be used as a normal working drone in high altitude areas.

To determine the number of SSA drones, under the premise that we know that the flying distance of the drone is 30 kilometers and the distance that ordinary human eyes can detect is 2 kilometers, we set each detector to a radius $A$, in which a $2 \mathrm{~km}$ circle is used instead. Based on 


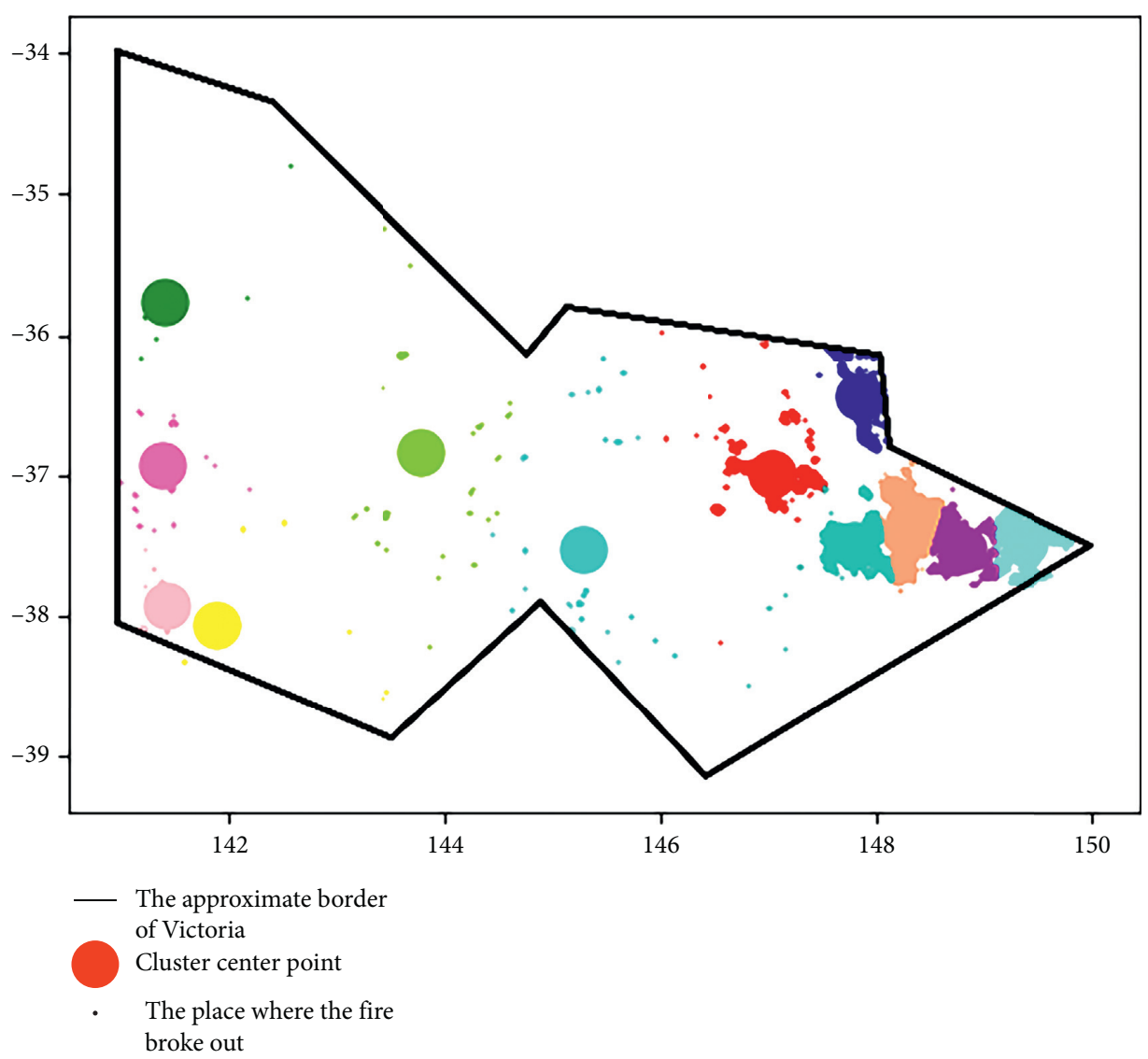

Figure 3: Cluster graph analysis.



Figure 4: Trend chart of drone coverage changes with altitude.

the human body's naked eye detection distance of $2 \mathrm{~km}$, within the effective working range of the EOC, at least 7 SSA orbits are required to achieve the purpose of complete coverage monitoring. At the same time, we use the idea of a greedy algorithm based on UAV performance to maximize the use of UAV flight capabilities. We 



- SSA effective detection range

- Repeater UAV coverage


- SSA effective detection range

- SSA effective detection range

— Repeater UAV coverage

- Repeater UAV coverage

Figure 5: Analysis of the number of drones.

determine the best path of SSA UAVs through iterative calculations [35] to determine the minimum number of SSA UAVs. We simplify the UAV flight path into a circle with the same center, as shown in Figure 6:

Through repeated calculations, the following conclusions are drawn:

(1) The SSA UAV can only fly half a circle on an orbit with a radius of 28 kilometers, and then the remaining range can fly one circle on an orbit with a radius of 4 kilometers. Currently, 4 drones are needed.
(2) The SSA UAV can only fly half a circle on an orbit with a radius of 24 kilometers. Then, the remaining range can fly two circles on an orbit with a radius of 4 kilometers or one circle on an orbit with a radius of 8 kilometers. At this time, 4 drones are needed.

(3) The SSA UAV can fly a circle in orbit with a radius of 20 kilometers and then needs to return to EOC immediately. At this time, 2 drones are required.

(4) The SSA UAV can fly one circle in orbit with a radius of 16 kilometers and then needs to return to EOC immediately. At this time, 2 drones are required. 


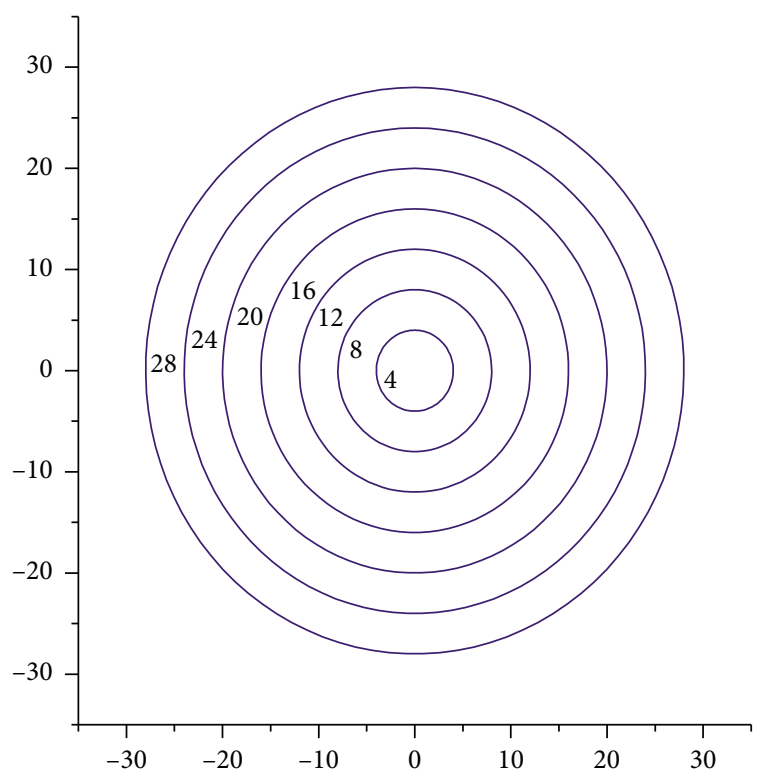

Figure 6: Concentric circle analysis diagram.

(5) The SSA UAV can fly one circle on an orbit with a radius of 12 kilometers; then, it can fly three times on an orbit with a radius of 4 kilometers and one flight on an orbit with a radius of 8 kilometers.

In summary, we do not need to purchase additional drones on orbits with a radius of 4 kilometers or a radius of 8 kilometers. At the same time, we also need to consider the SSA drone's rotation, so we choose twice the original number. We will add two SSA drones as backup in areas where severe fires have occurred for safety and comprehensive considerations.

In order to enable the government to respond to the fire in the shortest possible time, we combine the conclusions mentioned above to establish a final rapid emergency response model to help the government better deal with the fire. Based on the most optimal location, we can cover most of the fire spots. Simultaneously, due to different factors such as altitude, in order to conduct detailed analysis at different locations, we know the number of drone combinations required under zero-altitude aircraft through the previous analysis. Therefore, we analyze the fire points at different altitudes in Victoria based on zero altitudes.

Since the monitoring range of SSA UAVs is not affected by terrain factors, to determine the number of SSA UAVs, only the number of repeater drones is determined. We take the performance and economic conditions of drones as constraints and enumerate the number of SSA drones and the number of repeater drones required for each altitude segment. Thus, the relationship between the number of drones that need to be purchased at different heights in the city and the number of drones that need to be purchased in flat areas is obtained.

Terrain factors include urban areas and flat areas with no obstacles. Since the known communication signal ranges are 2 kilometers and 5 kilometers, respectively, we will discuss the number of repeater drones required in urban areas and flat areas based on the obtained altitude points. Using ArcGIS to draw a topographic map of Victoria, we can see that fire hazard area's altitude is between -15.9037 meters and 1981.95 meters. We set the height corresponding to the reference detection range to 0 meters. From the picture, we can see that there are mountains with higher elevations on the southeast side of Victoria, which are also the places with the highest frequency and intensity of fires. The western and central regions are relatively low, and fires' frequency and intensity are lower than those in the eastern region. In addition, the blue circle is the signal range of the repeater and should be cut with the red circle representing the effective working range of the EOC to achieve the effect of real-time reporting.

The direct drone's main function is to provide signal coverage, so we must consider that the farthest signal that the communication device can transmit in a flat, unobstructed area is 5 kilometers. We use interpolation to calculate from 0 meters above sea level and set the highest elevation to 2000 meters. We regard every 200 meters as a segment, so we choose 1800 meters and 2000 meters above sea level, which is the closest to 1981.95 for calculation.

Under the condition of 1800 meters above sea level, the communication distance of communication equipment in the barrier-free flat area is 5 kilometers, so we can place a set of communication equipment at the origin of EOC so that we can get a circle with EOC as the center and radius of 5 kilometers. We use this circle as our communication area.

From the most basic zero-height model, we can conclude that only three repeater drones need maximum coverage. Due to the increase in altitude, the number of direct drones required will have two situations: increase or unchanged. We consider the coverage of three repeater drones and four repeater drones.

We use python software to make the signal range that the three repeater drones can cover, as shown by the blue circle in Figure 7. Due to the increase in altitude, the repeater drone's signal range at this time is less than zero. At the same time, the signal receiving range and detection range of the detector must be considered.

The left panel of Figure 7 shows the range that the detector can detect when a signal is received. The red dot in the middle represents EOC, the small blue circle in the middle represents the signal coverage of EOC, and the red circle represents the effective detection range of EOC. Also, the large blue circle surrounding the small blue circle represents the detection range of the $U A V$, the green circle represents the detection and the maximum noninfluencing contact range of the carrier's radio; at this time, we arrange them into an equilateral triangle according to the principle of stability and maximum coverage. It can be seen from the figure that the situation is similar to the zero-altitude situation, which can save the economy from using it as much as possible while ensuring safety. So, we think this situation is acceptable.

Then, we consider the case of four drones in the right panel of Figure 7. We also use python to achieve the signal coverage of the four repeater drones and the range that the 

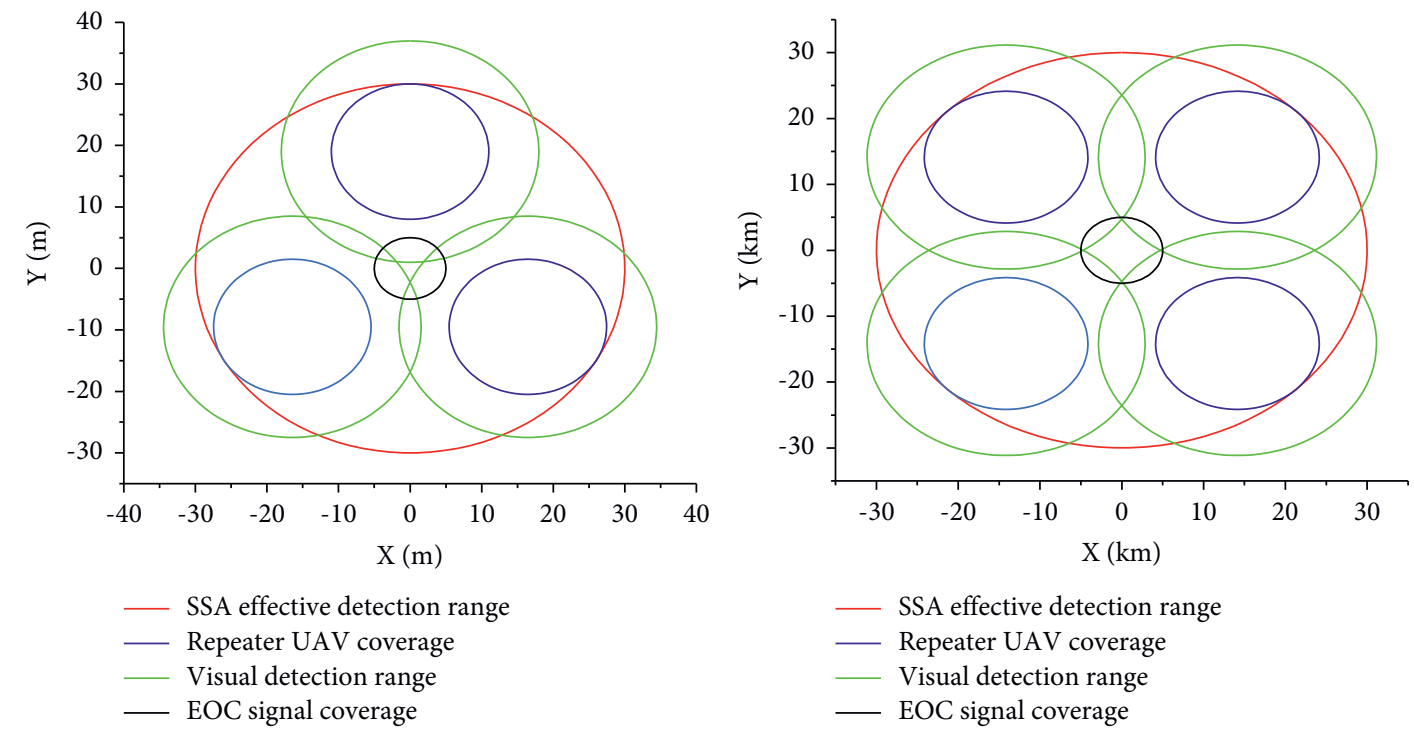

FiguRE 7: Three and four drones present a rectangular shape.

detector can detect when the signal is received. The meaning of each color is the same as the three repeater drones. We can see that the four drones are best arranged in a rectangle so that the maximum coverage can be guaranteed under the principle of economy.

In order to judge whether the economy will damage the detection range, we will start with the coverage of the UAV detection range. The red circle area represents the SSA effective detection range, while each blue circle represents a drone, and each green circle represents the visual observation range; in order to avoid dead corners to the greatest extent, we will actually select the number of UAVs in operation as twice the number in the image for cross patrol. Therefore, the detection range of drones is the product of area and the number of drone patrols.

Combining the above two situations and analyzing the data in Table 1, we can see that at 1800 meters, 3 repeater drones and 4 repeater drones can achieve good coverage. However for economic reasons, the coverage rate of four UAVs has limited growth compared with three, so we choose to use 3 repeater drones to work at this height. Since drone batteries need to be charged, we need to take turns to work. We chose to buy six drones which is twice as much as the image. For safety reasons, we should purchase two more repeater drones and two SSA drones.

As the altitude decreases, the direct drone coverage will increase, so the situation with an altitude of fewer than 1800 meters is better than the situation with an altitude of 1800 meters. So we bought 8 repeater drones and 14 repeater drones.

Then, we consider the situation of 2000 meters above sea level. At this time, the communication distance of the communication device is still 5 kilometers. Based on the consideration at an altitude of 1800 meters, we still consider three repeater drones and four repeater drones. When we use three drones, we use python to simulate the coverage of these three drones and the detectable range of the detector.
We can see that this situation is similar to 1800 meters, and the coverage area has not decreased much. At this time, we still need two spare drones. The number of drones we need to buy is 22. It contains 8 repeater drones and 14 SSA drones.

When we use four drones, we find it suitable for the signal coverage of the repeater drone and the detection range of the detector. Although the coverage is relatively comprehensive, the number of drones required is huge. Because we cluster more clusters, the total cost will also be higher. Moreover, in Figure 8, we can see that compared with three UAVs and four UAVs, there is not much difference in the detection range between the two; therefore, we still choose three UAVs for economic reasons. Based on the 2000-meter altitude situation discussion, we chose the coverage of 3 repeater drones, which means we need to purchase 8 repeater drones and 14 SSA drones.

Completed the combined analysis of the number of drones in the plane barrier-free area and the best position of the direct drone. Based on the analysis we have completed, we will continue to analyze the urban area's situation. The difference between an urban area and a flat barrier-free area is that the communication distance of people's communication equipment is different, and the urban area is reduced to 2 kilometers. At this time, we changed the signal coverage of EOC from 5 kilometers to 2 kilometers. At the same time, the scope of the green circle will be reduced.

We are still analyzing based on the zero-height model, starting with three drones. Based on the analysis results, we are still analyzing the situation at an altitude of 1,800 meters. In summary, whether it is 1800 meters above sea level or 2000 meters above sea level, we all need to buy 8 repeater drones and 14 SSA drones. The repeater drone position is shown in Figure 9, so that it forms a triangle that can cover to the greatest extent.

Since the altitude has risen to 1800 meters, according to our assumption, our repeater drone coverage is reduced by 9 kilometers, so the current coverage is 11 kilometers, which 
TABle 1: Comparison of coverage under different modes.

\begin{tabular}{lccccc}
\hline Patrol mode & SSA range & UAV range & Ratio of coverage & Visual range & Ratio of visual coverage \\
\hline Three drones & 2827.43 & 2280.78 & 0.8067 & 6107.256 & 2.16 \\
Four drones & 2827.43 & 2513.272 & 0.8889 & 7263.36 & 2.57 \\
\hline
\end{tabular}

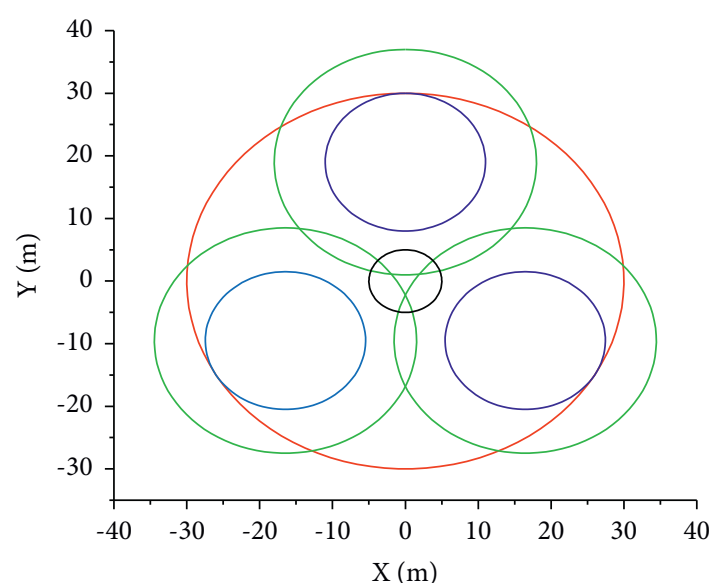

__ SSA effective detection range
Repeater UAV coverage
_ Visual detection range
_ EOC signal coverage

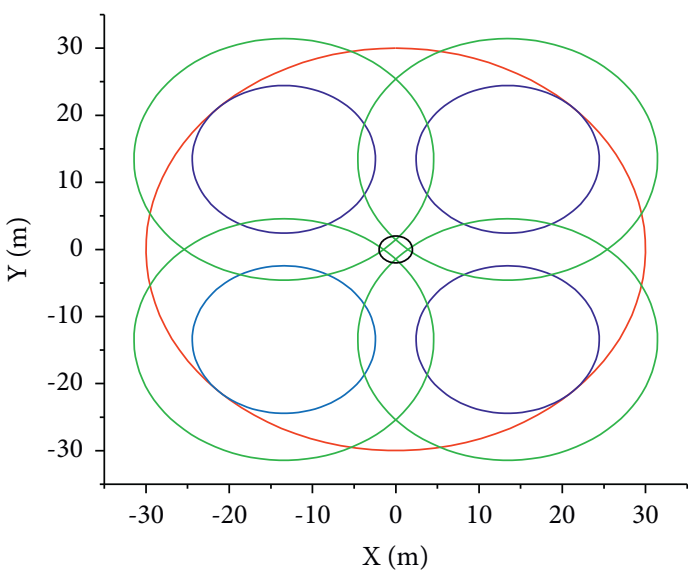

- SSA effective detection range

- Repeater UAV coverage

- Visual detection range

_ EOC signal coverage

FIgURE 8: Comparison of three drones and four drones.


Figure 9: Comparison of the detection of three drones and four drones at 1800 meters.

means that what we can do is a radius of 11 kilometers. It is engraved on the circle formed by the maximum flight distance of the SSA UAV. Since the signal transmission distance of the detector's device is 2 kilometers, we can get the widest communication coverage.
As can be seen from Figure 9, because the detection range of the equipment worn by the detector has been reduced by 3 kilometers, its coverage is minimal. Although economic principles can still be met, it is no longer possible to meet safety requirements. We need to add a repeater 
TABle 2: Comparison of coverage under different modes at $1800 \mathrm{~m}$ altitude.

\begin{tabular}{lccccc}
\hline Patrol mode & SSA range & UAV range & Ratio of coverage & Visual range & Ratio of visual coverage \\
\hline Three drones & 2827.43 & 2280.78 & 0.8067 & 6107.256 & 2.16 \\
Four drones & 2827.43 & 3619.1 & 1.28 & 6432 & 2.275 \\
\hline
\end{tabular}

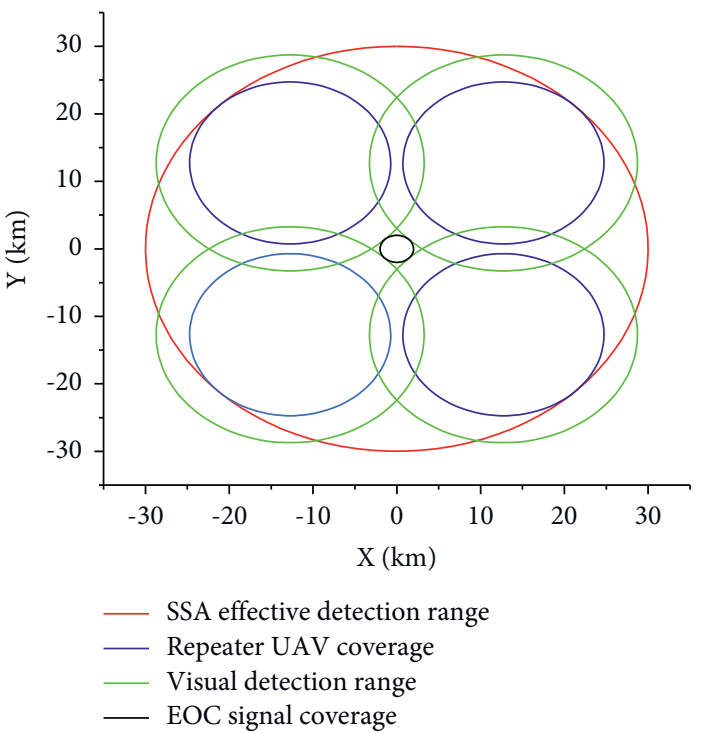

Figure 10: The drone presents the difference between a regular hexagon and a square.

drone, that is, 4 repeater drones, to cover this area. We still use the same method to make the corresponding situation.

After calculation in Table 2, the signal range covered by the four direct drones is the same as the signal range covered by the three direct drones in the zero-height model. We need to consider safety first and then decide on economics. Therefore, for urban areas, the solution of 4 straight drones is better than 3 straight drones.

Then, we have to consider the case of full coverage. In this case, we consider security and ignore economy. The purpose of this situation is to verify whether these 4 drones can meet the safety requirements. Since the inscribed circle cannot fill the red circle, we simplified the red circle to a regular hexagon. Its area is slightly reduced within a reasonable range. Its purpose is to meet the requirements of inscribed circles. The circle can better cover this number.

It can be seen from Figure 10 and Table 3 that only 7 circles can completely cover the red circle; the cost is high, it cannot meet the financial requirements, and the coverage area is wasteful. In this case, we can compare the situation of four direct drones, and we can find that the coverage of the four direct drones is not much smaller than this situation. Within a reasonable range, the coverage rate is high and economical.

In summary, at an altitude of 1800 meters, we selected 4 repeater drones for signal coverage. Currently, we still need 8 repeater drones and 14 SSA drones. When we choose four drones to work, the position is a circular inscribed rectangle.
The situation of two EOCs is more complicated; since there is already a basis for one EOC result, for larger clusters, we will introduce another EOC for consideration. From the approximate elevation, their average altitudes are $1600 \mathrm{~m}$, $1000 \mathrm{~m}$, and $600 \mathrm{~m}$, respectively. We consider the city and the countryside separately and first analyze the countryside. At 0 altitude, we combine the repeaters owned by the two EOCs at fixed points, and the following graph can be obtained in the left panel of Figure 11.

From this, we can find that the repeater drone has covered the detection range well, and we save one repeater compared to two independent EOCs.

For the case of $600 \mathrm{~m}$, we perform the same processing, and we can get the following graph in the right panel of Figure 11:

The conclusion is the same as that at 0 meters. Repeater drones also better cover the monitoring range.

It can be seen from Table 4 that the detection coverage of the five UAVs at zero altitude is very high, and the detection coverage decreases at $600 \mathrm{~m}$ altitude, but it is still about 0.8 , which meets the standard of real-time detection. Similarly, their ratio of visual coverage is greater than one, indicating that the conclusion is feasible.

We raise the altitude to $1000 \mathrm{~m}$ for research and adopt the same processing method, and we can get the following situation in the left panel of Figure 12:

It can be seen that the results are almost the same as the above two cases, so at this time, we think that for the case of two EOCs below 1000 meters above sea level, we only need 5 repeater drones for full coverage. 
TABLE 3: Comparison of coverage under different modes between a regular hexagon and a square.

\begin{tabular}{lccccc}
\hline Patrol mode & SSA range & UAV range & Ratio of coverage & Visual range & Ratio of visual coverage \\
\hline Regular hexagon & 2827.43 & 4398.226 & 1.55 & 8620.528 & 3.04 \\
Square & 2827.43 & 3619.1 & 1.28 & 6432 & 2.275 \\
\hline
\end{tabular}
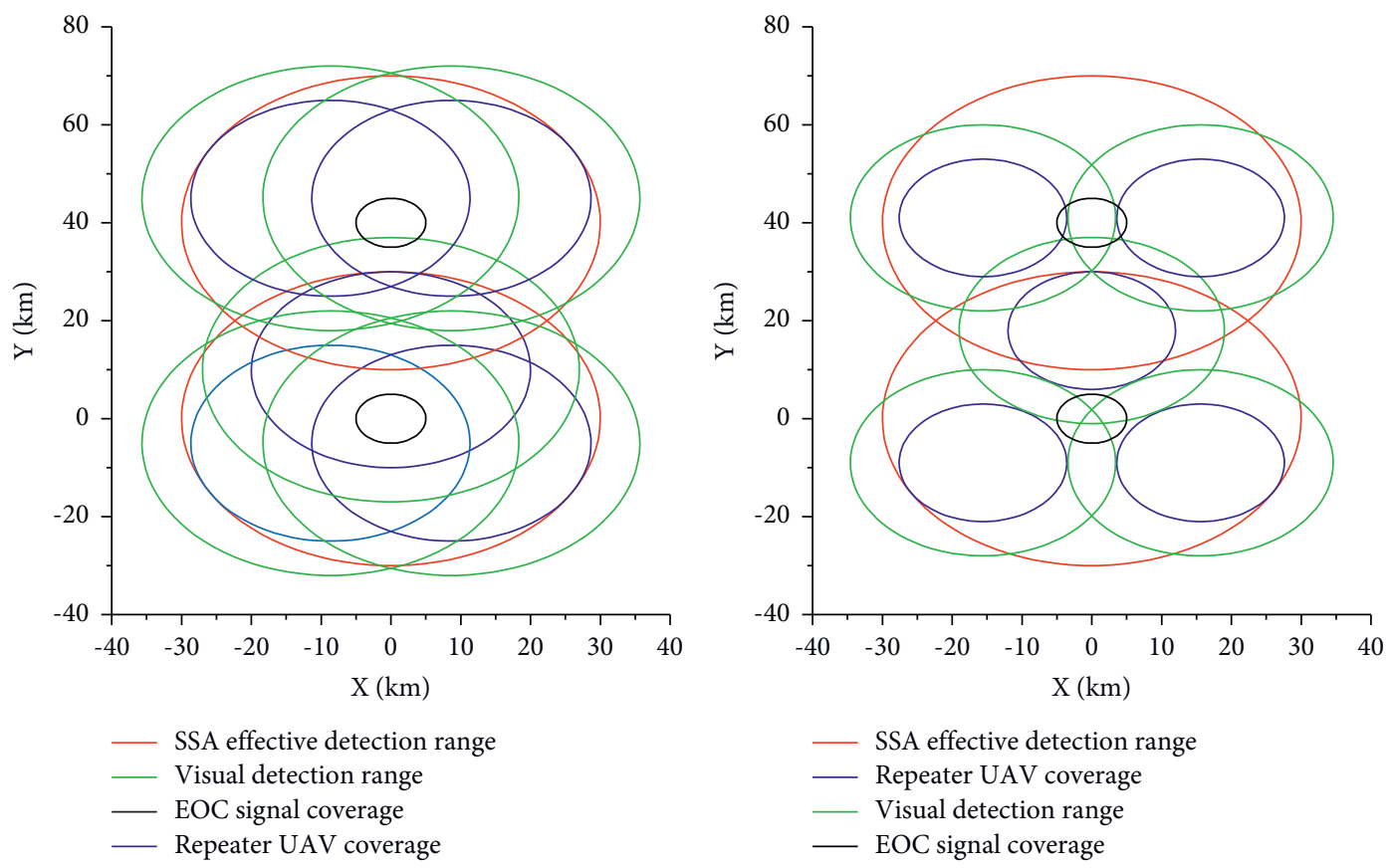

Figure 11: Two EOCs in the village at zero altitude and 600 meters.

TABLE 4: Comparison of coverage under different modes between zero attitude and 600 meters.

\begin{tabular}{lccccc}
\hline Patrol mode & SSA range & UAV range & Ratio of coverage & Visual range & Ratio of visual coverage \\
\hline Zero attitude & 5654.86 & 12566.37 & 2.22 & 19634.95 & 3.47 \\
600 meters & 5654.86 & 4523.89 & 0.80 & 9079.2 & 1.606 \\
\hline
\end{tabular}
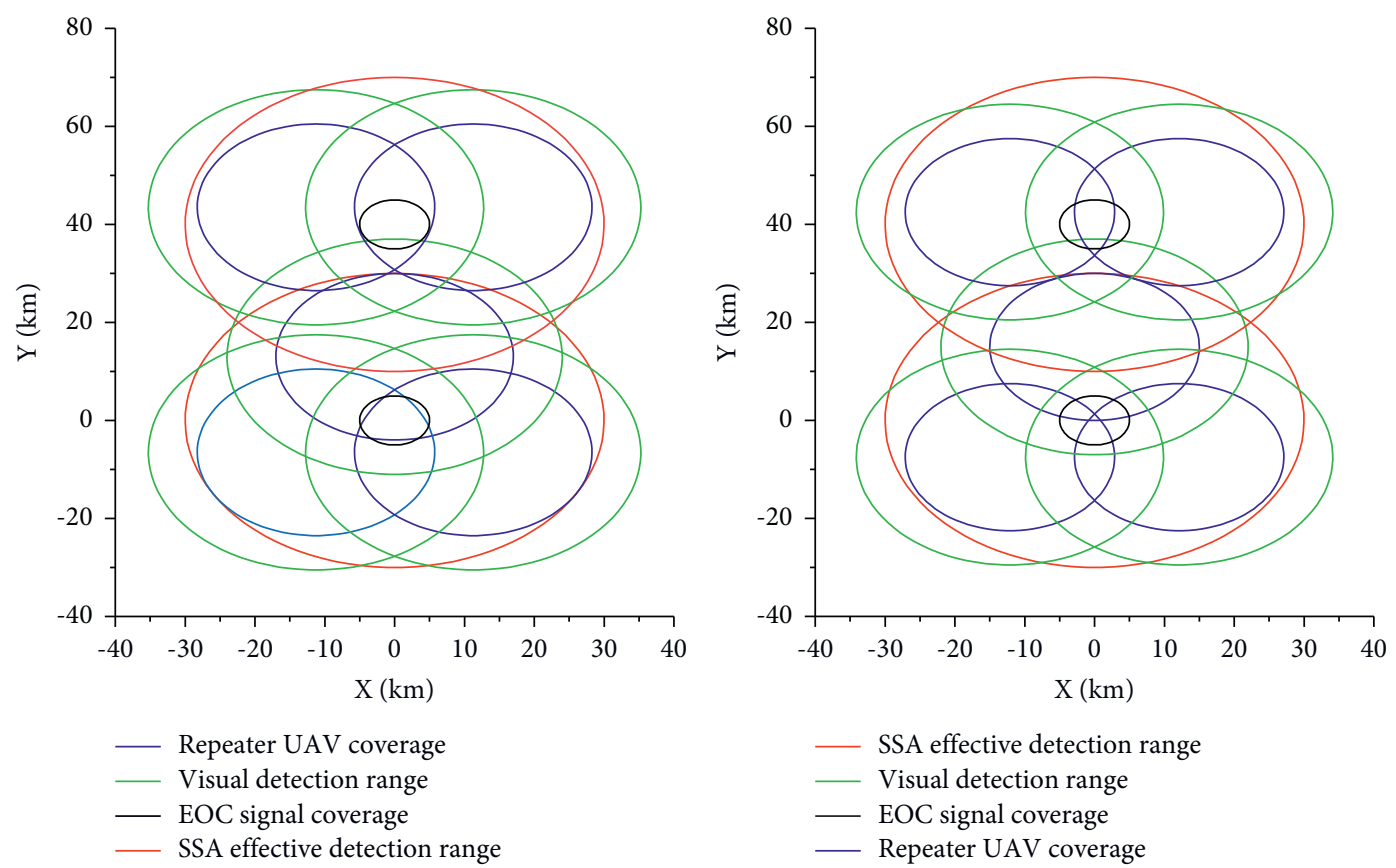

FIgURE 12: Detection of two EOCs in the countryside at 1000 meters and 1600 meters. 
TABle 5: Comparison of coverage under different modes between 1000 meters and 1600 meters.

\begin{tabular}{lccccc}
\hline Patrol mode (meters) & SSA range & UAV range & Ratio of coverage & Visual range & Ratio of visual coverage \\
\hline 1000 & 5654.86 & 9079.2 & 1.61 & 15205.3 & 2.69 \\
1600 & 5654.86 & 7068.5 & 1.25 & 12566.37 & 2.22 \\
\hline
\end{tabular}
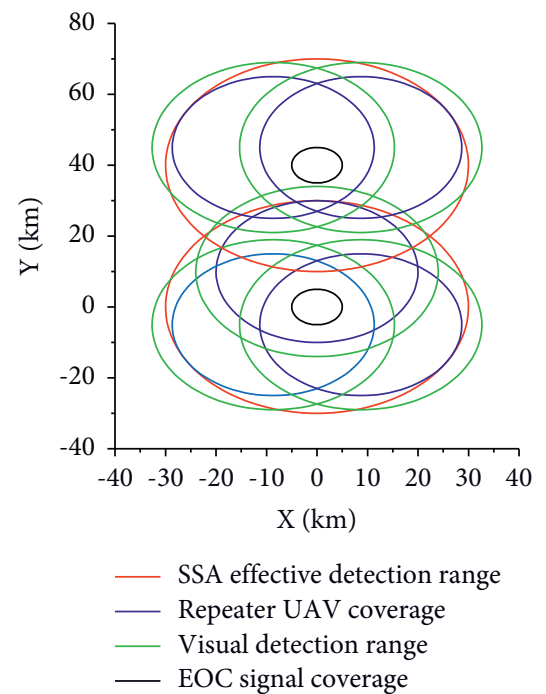

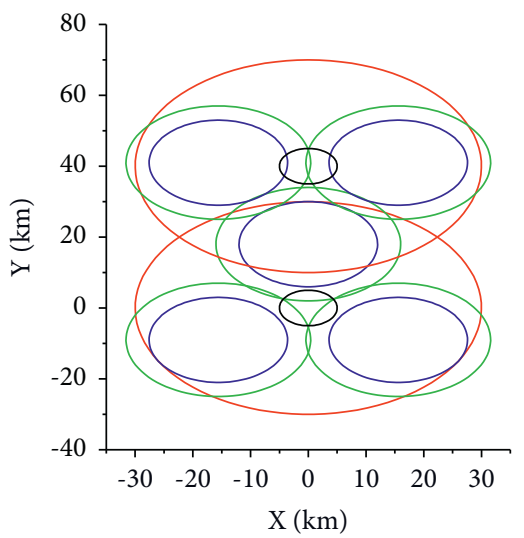

— SSA effective detection range

- Repeater UAV coverage

- Visual detection range

- EOC signal coverage

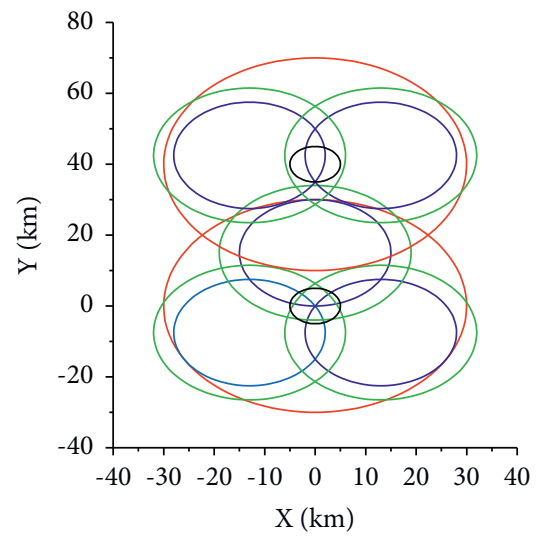

SSA effective detection range
Repeater UAV coverage
- Visual detection range
EOC signal coverage

Figure 13: Detection of two EOCs in the city at zero altitude, 600 meters, and 1000 meters.

TABlE 6: Comparison of coverage under different modes in the city.

\begin{tabular}{lccccc}
\hline Patrol mode & SSA range & UAV range & Ratio of coverage & Visual range & Ratio of visual coverage \\
\hline Zero attitude & 5654.86 & 12566.37 & 2.22 & 15205.3 & 2.69 \\
600 meters & 5654.86 & 4523.89 & 0.80 & 6157.5 & 1.09 \\
1000 meters & 5654.86 & 7068.5 & 1.25 & 9079.2 & 1.61 \\
\hline
\end{tabular}

Then, we will discuss the case where the altitude is 1600 meters, as shown in the right panel of Figure 12.

Through the data of Table 5 and Figure 12, it can be seen that the five direct release UAVs cannot fully cover the area, and the loopholes at the upper and lower edges of the area are relatively large. However, when two UAVs work alternately, their coverage has a good value, which can fully cover the detection range. Therefore, we can use five repeater drones to detect the heights of these two altitudes.

Then, we will consider the city's situation; its influence on the signal is similar to the situation of an EOC we are discussing. We still consider from the 0 altitude, as shown in the left panel of Figure 13.

From Figure 13 and Table 6, we can see that in the case of zero altitude and $1000 \mathrm{~m}$, five repeated drones in each group are divided into two groups, and the coverage of simultaneous detection is greater than one. In the case of $600 \mathrm{~m}$, the value of ratio of coverage is 0.8 . In rural areas, due to the lack of buildings and open vision, the coverage is less than one, In case of fire, the smoke and fire precursor can also be detected by other UAVs, and the economic loss will not be great; however, due to the dense population and many complex buildings in the city, if the fire is not detected initially, it will cause heavy casualties and property losses. Therefore, we will increase the number of UAVs in each group at an altitude of $600 \mathrm{~m}$.

\section{Conclusion}

5.1. For the Urban Area. For areas where the cluster is small, and the altitude is less than or equal to 1600 , we use one EOC for coverage. At this time, it corresponds to 14 SSA drones and 8 repeater drones. One SSA UAV and two repeater UAVs are standby aircraft.

For areas where the cluster is small, and the altitude is greater than or equal to 1800 , we will still use one EOC for coverage. At this time, 14 SSA drones and 8 repeater drones should be used. Here, we only reserve 2 SSA drones and no repeater drones, and all repeater drones will be put into operation.

When the cluster is large, and the altitude is less than or equal to 600 meters, we can use two EOCs for coverage. At this time, 12 repeater drones and 26 SSA drones are required. Among them, there are 2 SSA drones in total. The man-machine and 2 repeater drones are used as backup machines.

When the cluster is large, and the altitude is greater than or equal to 1000 meters, we use two EOCs for coverage. At this time, each EOC needs to be considered separately because if the two are used as a whole, full coverage will not be possible. At this time, 10 repeater UAVs and 26 SSA UAVs are required, of which 2 SSA UAVs will be used as backup aircraft, and all repeater UAVs will be put into operation. 
5.2. For the Countryside. For areas with small clusters, regardless of altitude, we use one EOC for coverage. At this time, 14 SSA UAVs and 8 repeater UAVs are working. For the sake of consistency, 2 SSA UAVs and 2 Repeater UAVs are standby aircraft.

For areas with large clusters, when the altitude is less than or equal to 1000, we use two EOCs for coverage, and the two EOCs can work in coordination. At this time, 10 repeater drones and 26 SSA drones are needed. Among them, a total of 2 SSA drones and 2 repeater drones are used as backup aircraft.

When the altitude is greater than or equal to 1600 , we will use two EOCs for independent work for areas with large clusters. At this time, 10 repeater drones and 26 SSA drones are required, of which 2 SSAs are unmanned. The aircraft will be used as backup aircraft, and all repeater drones will be put into operation.

The continuous development of technology will improve the existing situation and solve problems better [36]. We have reason to believe that the leap of UAV technology will bring sustainable and long-term and profound impact on Australian mountain fire control.

5.3. For the Potential Applications. Because our model simplifies many variables, our model is conducive to the application of similar situations in the future. Similarly, there is an instrument with a general control center that controls the surrounding area, Therefore, our model can be used in the dynamic process of projects that use surrounding instruments to work, including the robot temperature detection in China during the epidemic, that is, a general control center controls the movement of the temperature measurement robot in the airport or open area, so that the temperature measurement robot can detect the temperature within the range allowed by the control center, Or, similar to the dynamic change process of WiFi signals connected by mobile phones and other tools within the influence range of a router, these can be used in our model.

5.4. For the Weaknesses. The shortcomings of this paper are mainly divided into two cases.

The first is a small probability event. For example, the dust caused by mountain fire will short circuit the UAV and affect the vision. The EOC signal is not invariable within this range, and the signal reception will change with the distance between the UAV and the EOC, or there are a large number of trees in the forest area with different heights; therefore, the motion of UAV is not the circular uniform motion in the perfect form we assume.

Secondly, our model cannot analyze the possible dead corners of UAV detection. During the movement of UAV, if there is a mountain fire just after the UAV leaves this movement range, how should we solve it? Therefore, this is the limitation of our model. The solution is to increase the number of UAVs, but it will lead to more economic consumption and inefficient detection. Moreover, since the model assumes a perfect situation, rashly increasing the number of UAVs will also lead to more problems due to
UAV operation. Therefore, how to prevent or reduce the frequency of detection blind areas is the biggest limitation of the current model.

\section{Data Availability}

We can obtain data and dynamic image from https://mail. qhu.edu.cn/coremail/XT5/jsp/download.jsp?share_link=ED 5B238CE0F34B6EBE26022D434B8A6F\&uid $=1800310027 \%$ 40qhu.edu.cn. The data link password is iwTC.

\section{Conflicts of Interest}

The authors declare that there are no conflicts of interest regarding the publication of this paper.

\section{Acknowledgments}

This work was supported by the following projects: National Natural Science Foundation of China (nos. 51779272, 51869025, 51769028, 51909129, and 51868066), National Key R\&D Program of China (no. 2017YFC0404303), Qinghai Department of Science \& Technology Projects (no. 2021-SF134), National Key Laboratory Project for Water Sand Science and Water and Hydropower Engineering, Tsinghua University (Grant no.sklhse-2021-B-04).

\section{References}

[1] W. Zhang, Q. Wang, and R. Wang, "Research progress of Eucalyptus forest fire in Australia," World Forestry Research, vol. 1, no. 10, pp. 1-10, 2021.

[2] J. C. Ditto, M. He, T. N. Hass-Mitchell et al., "Atmospheric evolution of emissions from a boreal forest fire: The formation of highly functionalized oxygen-, nitrogen-, and sulfur-containing organic compounds," Atmospheric Chemistry and Physics, vol. 21, no. 1, pp. 255-267, 2021.

[3] S. Wei, R. Sheng, B. Luo, and X. Li, "Study on the occurrence law of forest fire under the background of climate change," Forestry and Environmental Science, vol. 36, no. 2, pp. 133$143,2020$.

[4] S. Oliveira, J. M. C. Pereira, J. San-Miguel-Ayanz, and L. Lourenço, "Exploring the spatial patterns of fire density in southern europe using geographically weighted regression," Applied Geography, vol. 51, no. 2, pp. 143-157, 2014.

[5] M. Zhu and R. Fegely, "Overview of Australian forestry/forest products trade and investment opportunities," Wood industry, vol. 30, no. 1, pp. 5-9, 2016.

[6] P. A. Rodriguez, W. J. Geckle, J. D. Barton et al., "An emergency response UAV surveillance system," in Annual Symposium Proceedings. AMIA Symposium, Bethesda, MD, USA, Febuary 2006.

[7] Z. Jian, J. Lu, and J. Guo, "Optimal dispatch model of fire extinguishing equipment for electric grid under wild fire disaster with multiple fire points," Power System Technology, vol. 42, no. 7, pp. 2324-2330, 2018.

[8] F. Ding, Q. Li, and L. Jin, "Experience and practice of the emergency operations center, Chinese center for disease control and prevention: a case study of response to the H7N9 outbreak," Infectious Diseases of Poverty, vol. 10, no. 1, 2021.

[9] T. Ju, Research on Application of UAV Detection Technology for Accidents and Disasters in Dangerous Places, South China University of Technology, Guanzhou, China, 2017. 
[10] Y. Zhang, M. Huang, and J. Yang, "Comparison of FM broadcast monitoring coverage of VHF/UHF fixed monitoring stations," China Radio, vol. 2020, no. 11, pp. 44-47, 2020.

[11] C. Yang, "Design of microwave front end for UAV relay TT \& C link," Telecommunication Technology, vol. 60, no. 5, pp. 524-528, 2020.

[12] X. Zhang, Y. Hu, W. Li, Q. Pang, and G. Yuan, "Multi UAV fire fighting task planning based on improved artificial colony algorithm," Journal of China Inertial Technology, vol. 28, no. 4, pp. 528-536, 2020.

[13] K. Deng, Z. Lian, and D. Zhou, "Task allocation of Multiunmanned aerial vehicle based on improved quantum particle swarm optimization," Command Control \& Simulation, vol. 40, no. 5, pp. 32-36, 2018.

[14] H. Kurdi, J. How, and G. Bautista, "Bio-inspired algorithm for task allocation in multi UAV search and rescue missions," in Proceedings of the AIAA Guidance, Navigation and Control Conference, San Diego, CA, USA, January 2016.

[15] S. Yong, "Application advantages and Optimization Countermeasures of UAV system in forest fire prevention," Rural Science and Technology, vol. 11, no. 33, pp. 114-115, 2020.

[16] C. S. Reddy, N. G. Bird, S. Sreelakshmi et al., "Identification and characterization of spatio-temporal hotspots of forest fires in South Asia," Environmental Monitoring and Assessment, vol. 191, no. S3, 791 pages, 2020.

[17] J. G. Cawson and T. J. Duff, "Forest fuel bed ignitability under marginal fire weather conditions in Eucalyptus forests," International Journal of Wildland Fire, vol. 28, no. 3, pp. 198204, 2019.

[18] M. Almeidam, D. X. Viegas, A. I. Miranda, and V. Reva, "Effect of particle orientation and of flow velocity on the combustibility of Pinuspinaster and Eucalyptus globulus firebrand material," International Journal of Wildland Fire, vol. 20, no. 8, pp. 946-962, 2012.

[19] M. Ren, J. Zhang, L. Khoukhi, H. Labiod, and V. Vèque, "A review of clustering algorithms in VANETs," Annals of Telecommunications, pp. 1-23, 2021.

[20] J. Yang and C. Zhao, "Review of $K$-means clustering algorithm," Computer Engineering and Application, vol. 55, no. 23, pp. 7-14, 2019.

[21] A. K. Jain, M. N. Murty, and P. J. Flynn, "Data clustering," ACM Computing Surveys, vol. 31, no. 3, pp. 264-323, 1999.

[22] M. Hai, S. Zhang, and Y. Ma, "A survey of clustering algorithms in distributed environment," Computer Application Research, vol. 30, no. 9, pp. 2561-2564, 2013.

[23] S. Chakraborty and S. Das, " $k$-means clustering with a new divergence-based distance metric: Convergence and performance analysis," Pattern Recognition Letters, vol. 100, pp. 67-73, 2017.

[24] J. Dong, "Comparison and analysis of dynamic programming algorithm and greedy algorithm," Software Guide, vol. 2008, no. 2, pp. 129-130, 2008.

[25] H. Fang, Z. He, F. Shan, M. Tie, X. Zhang, and J. Sun, "Efficient mesh deformation using radial basis functions with a grouping-circular-based greedy algorithm," Journal of Computational Physics, vol. 433, no. 1, 2021.

[26] X. Wang, Computer Algorithm Design and Analysis, Electronic Industry Press, Beijing, China, 5th edition, 2018.

[27] W. Huang, M. Jia, and L. Huang, "Research on map reduce load balancing of parallel random sampling greedy algorithm partition," Modern Electronic Technology, vol. 43, no. 16, pp. 170-173, 2020.
[28] Z. Yang, Y. LV, and C. Cao, "Application of greedy algorithm in scientific research instrument sharing system," Automation Technology and Application, vol. 39, no. 11, pp. 169-173, 2020.

[29] J. Wang, X. Ma, J. Wu, and Y. Dong, "Optimization models based on GM $(1,1)$ and seasonal fluctuation for electricity demand forecasting," International Journal of Electrical Power \& Energy Systems, vol. 43, no. 1, pp. 109-117, 2012.

[30] T. Yuan, J. Yuan, and Q. Chao, "Study on comprehensive model for medium and long term load forecasting of power system," Protection and Control of Power System, vol. 2012, no. 14, pp. 143-146, 2012.

[31] C. Zhao and M. Fang, "Design and implementation of logistics distribution system based on greedy algorithm," Software Engineering, vol. 23, no. 5, pp. 21-23, 2020.

[32] K. J. Anil, "Data clustering: 50 years beyond K-means," Pattern Recognition Letters, vol. 31, no. 8, pp. 651-666, 2010.

[33] S. Kavita, "Review: Study on simple $k$ mean and modified $K$ mean clustering technique," International Journal of Computer Science Engineering and Technology, vol. 6, no. 7, pp. 279-281, 2016.

[34] X. Dai and F. Chen, "Multi-UAV cooperative search on region division and path planning," Acta Aeronautica Et Astronautica Sinica, vol. 41, no. S1, pp. 149-156, 2020.

[35] W. Hu, "Improved hierarchical $K$-means clustering algorithm," Computer Engineering and Application, vol. 49, no. 2, pp. 157-159, 2013.

[36] Y. Wu, L. Tian, M. Rubinato et al., "A new parallel framework of SPH-SWE for dam break simulation based on OpenMP," Water, vol. 12, no. 5, 1395 pages, 2020. 